\title{
Milrinone in heart failure Effects on exercise haemodynamics during short term treatment
}

\author{
A D TIMMIS, P SMYTH, D E JEWITT \\ From the Department of Cardiology, King's College Hospital, London
}

SUMMARY The effects of milrinone, a new bipyridine inotropic agent, on the haemodynamic responses to treadmill exercise were studied in 12 patients with congestive heart failure. Four weeks' treatment with milrinone $20 \mathrm{mg}$ daily produced an improvement in left ventricular function during exercise as reflected by significant increments in cardiac index and stroke volume index without change in pulmonary capillary wedge pressure. Systemic oxygen consumption, measured at submaximal exercise, also increased suggesting that the drug induced rise in stroke output was associated with improved skeletal muscle perfusion. Maximum exercise capacity increased. Importantly, the beneficial effects of milrinone on exercise haemodynamics and exercise tolerance were sustained throughout the four week treatment period. No drug related side effects occurred. After treatment with milrinone was stopped left ventricular function deteriorated to a level slightly, but significantly, worse than that before treatment.

These observations indicate a potentially useful role for milrinone in treating heart failure.

Although treatment with diuretics effectively controls the congestive manifestations of heart failure, the search for agents that provide sustained improvement in left ventricular function has been disappointing. The value of digitalis remains controversial, ${ }^{1}$ and despite the beneficial acute effects of vasodilators and beta adrenoceptor stimulants attenuation of responsiveness to these agents frequently occurs. ${ }^{2-4}$. The introduction of amrinone, therefore (a non-adrenergic inotropic agent) generated considerable interest. Amrinone is a bipyridine compound that improves left ventricular function in the short term, ${ }^{56}$ though long term benefit has not been confirmed in controlled studies. ${ }^{7}$ The clinical usefulness of amrinone is further limited by its toxic properties, which have led to thrombocytopenia and gastrointestinal intolerance in many patients treated with the drug. ${ }^{8}$ More recently, a second bipyridine compound, milrinone, has been developed. Milrinone has greater positive inotropic potency than amrinone in experimental pre-

Requests for reprints to Dr A D Timmis, Department of Cardiology, Guy's Hospital, London SE1 9RT.

Accepted for publication 19 March 1985 parations ${ }^{9}$ and, importantly, does not appear to share the toxic properties of its parent compound.10-12 In patients with heart failure, milrinone produces a prompt improvement in left ventricular function through a combination of positive inotropism and vasodilatation; long term benefit in terms of improved exercise tolerance has also been reported. ${ }^{10-12}$ Placebo treatment, however, can have similar beneficial effects on exercise tolerance in heart failure, ${ }^{13}$ and for this reason measurement of the haemodynamic responses to exercise is necessary to confirm sustained drug efficacy. In this report we describe the effects of milrinone on haemodynamic responses to treadmill exercise during treatment of patients with congestive heart failure.

\section{Patients and methods}

PATIENT SELECTION

The 12 patients included in this short term evaluation of milrinone had all undergone an acute haemodynamic study with the same drug at least three days previously (range 3-16 days). Table 1 summarises the patients' characteristics and selection criteria, which have been previously described. ${ }^{14}$ Written 
Table 1 Patient characteristics

\begin{tabular}{lllllll}
\hline Case No & Sex/age $(\boldsymbol{r})$ & Diagnosis & NYHA class & EF (\%) & LVEDP $(\mathrm{mm}$ Hg) & Daily frusemide dosage (mg) \\
\hline 1 & M/39 & CAD & III & 10 & 31 & 120 \\
2 & M/55 & CAD & III & 27 & 16 & 250 \\
3 & M/45 & CAD & II & 44 & 24 & 80 \\
4 & M/54 & CAD & III & 13 & 23 & 80 \\
5 & M/64 & CAD & III & NA & 17 & 80 \\
6 & M/58 & CCM & III & 19 & 28 & 80 \\
7 & F/50 & CCM & II & 39 & 8 & 80 \\
8 & M/31 & CCM & III & 22 & 14 & 160 \\
9 & M/60 & CAD & III & 21 & 24 & 120 \\
10 & F/59 & CCM & III & NA & 25 & 8 \\
11 & M/53 & CCM & III & 23 & 15 & 120 \\
12 & M/64 & CAD & III & 10 & 15 & \\
\hline
\end{tabular}

CAD, coronary artery disease; CCM, congestive cardiomyopathy; EF, angiographic ejection fraction (not available (NA) in two cases); LVEDP, left ventricular end diastolic pressure (measured in the supine position).

informed consent was obtained from each patient before inclusion in the study, which had been approved by the hospital ethical committee.

\section{STUDY PROTOCOL}

All vasodilator treatment was discontinued at least a week before the study. Digoxin (four patients) and diuretic treatment were continued throughout the 30 days of the study without alteration in dosage. Exercise studies were always performed after fasting late in the afternoon about four to five hours after the midday milrinone capsule. The daily dose of diuretics and other regular medications were not withheld. The protocol was for a total of five exercise tests in each patient. The first, a preliminary test, permitted an assessment of maximal exercise capacity and was performed without invasive haemodynamic measurements. On day 1 of the study control haemodynamic data during exercise were obtained, after which treatment with oral milrinone $20 \mathrm{mg}$ daily divided into four doses was started. Further exercise tests were performed after two days and four weeks' of treatment-days 3 and 28 respectively. After day 28 milrinone was withdrawn, and two days later (day 30) the final exercise test was performed. Ten patients completed the protocol. One patient was withdrawn from the study after day 3 because of a transient cerebral ischaemic attack, which was not thought to be drug related. Another patient did not undergo the final exercise test after drug withdrawal on day 30 .

The patients were treated in hospital for the first three days of the study, after which outpatient follow up, with weekly review, continued until the last three days of the study when they were readmitted. At each follow up visit inquiries were made about drug related side effects, and the patients were supplied with 28 milrinone capsules (one week's supply). Compliance was confirmed by capsule counting. Blood samples for platelet estimation were obtained before exposure to milrinone and again after four weeks treatment (day 28).

\section{EXERCISE TESTS}

Heart rate was monitored from a precordial lead. Arterial blood pressure was recorded from a cannula placed in the radial artery. A balloon tipped thermodilution catheter placed in the pulmonary artery was used to monitor pulmonary capillary wedge pressure and cardiac output (measured in duplicate using $10 \mathrm{ml}$ of iced saline). All pressures were measured in the upright posture with reference to the sternal angle. This underestimated true left atrial pressure but ensured a constant zero level for all exercise tests. Arterial and mixed venous samples for gas analysis were drawn from the radial artery and the pulmonary artery respectively within one minute of the cardiac output measurements. Blood oxygen content, arteriovenous oxygen difference, systemic oxygen consumption index, and derived haemodynamic variables were calculated using standard formulae.

All patients were exercised on a treadmill. The Bruce protocol was used in seven cases but in the remainder, all of whom were more severely symptomatic, the less demanding Naughton protocol was used. The same exercise protocol was used throughout the study in individual patients. After maximal exercise capacity had been established in the preliminary test an arbitrary $70-80 \%$ submaximal level was calculated. In all subsequent exercise tests haemodynamic measurements were made at rest, after two minutes of exercise, at submaximal exercise, and at maximal exercise. A final set of measurements was made during recovery, one minute after maximal exercise. Blood samples for gas analysis were drawn within a minute of the submaximal haemodynamic measurements, shortly before maximal exercise. Note that in individual patients the same level of submaximal exercise was used in each test to provide a directly comparable set of data.

STATISTICAL ANALYSIS

The purpose of the statistical analysis was to detect a variation in the response to exercise over the 30 days 
Table 2 Haemodynamic responses to milrinone during exercise in 12 patients with congestive heart failure. Values are mean (SEM)

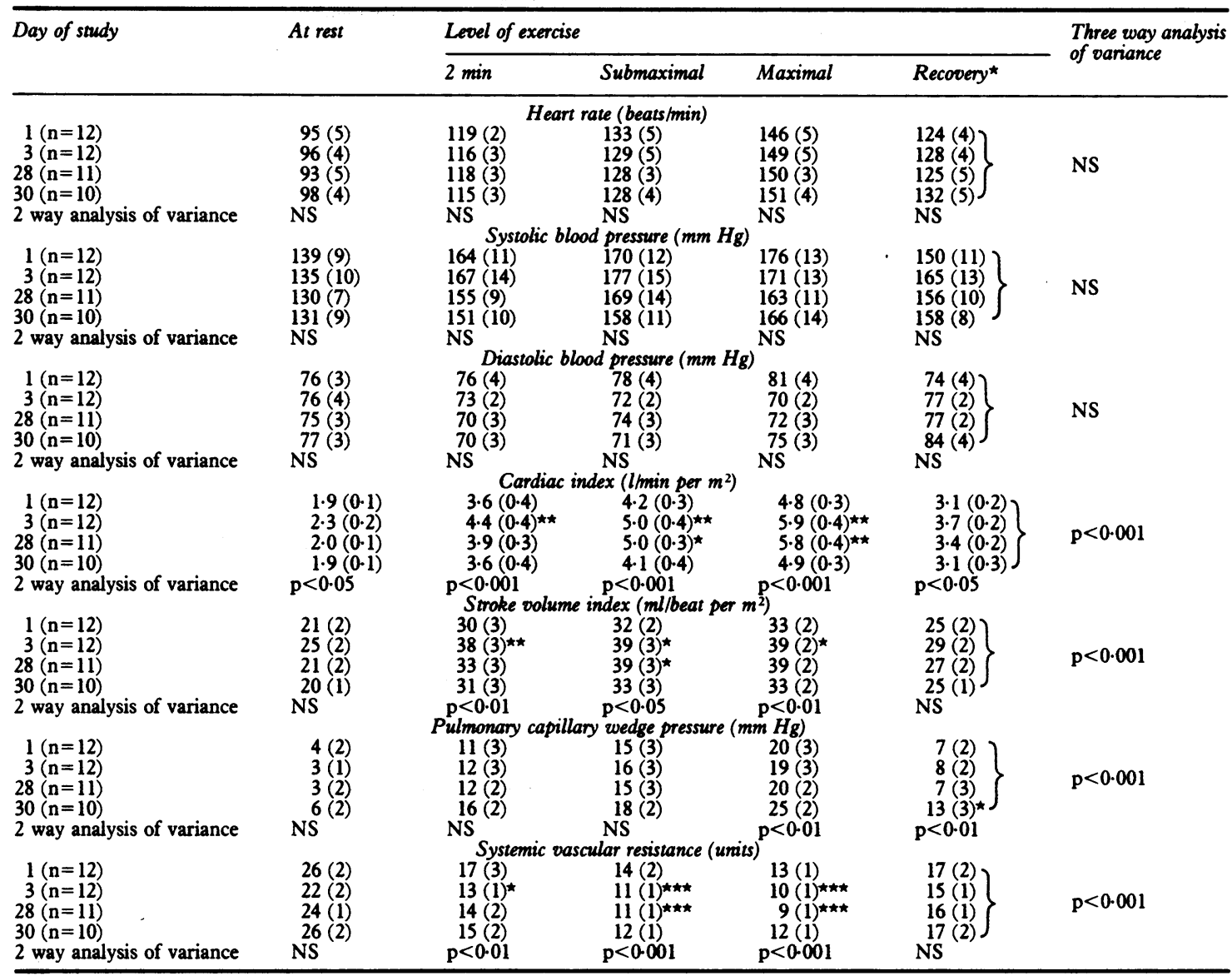

^One minute after peak exercise.

Statistical differences by 3 way and 2 way analyses of variance-significance of individual changes with respect to day $1:{ }^{\star} p<0.05,{ }^{\star} p<0.01$, $\star \star \star p<0.001$.

of the study. A three way analysis of variance was used to detect any variation in the haemodynamic measurements between the study days corrected for time. When a significant difference $(p<0.05)$ between days was found a two way analysis of variance was used to investigate the variation at each time point during the exercise period. Finally, a modified $t$ test, by the Bonferonni method, was used to detect the source of the differences at those times, where significant variation was present. ${ }^{15}$

Results are expressed as mean (SEM).

\section{Results}

\section{HAEMODYNAMIC RESPONSES}

Table 2 summarises the haemodynamic responses to treadmill exercise during the course of the study. The
Figure shows the effects of milrinone on stroke volume index and pulmonary capillary wedge pressure during exercise.

Control (day 1)-Despite the pronounced depression of resting cardiac index and stroke volume index before milrinone treatment, the pulmonary capillary wedge pressure (measured in the upright position) was normal owing to the influence of diuretic treatment. During exercise, however, the pulmonary capillary wedge pressure increased rapidly to 20(3) $\mathrm{mm} \mathrm{Hg}$ at peak exercise, though peak responses for cardiac index $\left(4.8(0.3) 1 / \mathrm{min}\right.$ per $\left.\mathrm{m}^{2}\right)$ and stroke volume index $\left(33(2) \mathrm{ml} /\right.$ beat per $\left.\mathrm{m}^{2}\right)$ were severely attenuated. Systemic oxygen consumption at submaximal exercise was only $56.7(5.4) \mathrm{ml} / \mathrm{min}$ per $\mathrm{kg}$ (Table $3)$. In all cases it was the early onset of fatigue and dyspnoea that limited exericise tolerance $(6.9(0.8)$ 
Table 3 Changes in systemic oxygen utilisation at submaximal and maximal exercise capacity. Values are mean (SEM)

\begin{tabular}{|c|c|c|c|c|c|}
\hline & Day 1 & Day 3 & Day 28 & Day 30 & $\begin{array}{l}\text { Two way analysis } \\
\text { of variance }\end{array}$ \\
\hline $\begin{array}{l}\text { Arteriovenous oxygen difference }(\mathrm{vol} / 100 \mathrm{ml}) \\
\text { Systemic oxygen consumption index }\end{array}$ & $13.1(0.7)$ & $13.4(0.6)$ & $13.6(0.7)$ & $13.8(0.7)$ & NS \\
\hline $\begin{array}{l}(\mathrm{ml} / \mathrm{min} \text { per } \mathrm{kg}) \\
\text { Maximal exercise capacity (min) }\end{array}$ & $\begin{array}{r}56.7(5.4) \\
6.9(0.8)\end{array}$ & $\begin{array}{r}67.8(6.3) \\
8.4(0.9)\end{array}$ & $\begin{array}{r}68.2(5.8) \\
8.9(0.9)\end{array}$ & $\begin{array}{r}58.5(6.3) \\
8.4(1.0)\end{array}$ & $\begin{array}{l}p<0.05 \\
p<0.001\end{array}$ \\
\hline
\end{tabular}

minutes; Table 3). Heart rate and blood pressure rose normally with exercise and systemic vascular resistance declined.

After milrinone (days 3 and 28)-Milrinone produced a small increase in resting cardiac index compared with control values but did not significantly influence the resting data in other respects. Exercise responses, however, showed important differences. Thus by day 3 of the study both cardiac index and stroke volume index were significantly higher than control values at every level of exercise. This improvement in the output response to exercise was sustained, and the measurements on day 28 were similar (Fig.). A sustained reduction in exercise systemic vascular resistance also occurred during milrinone treatment, though the changes in this variable were small. The arteriovenous oxygen difference at sub-

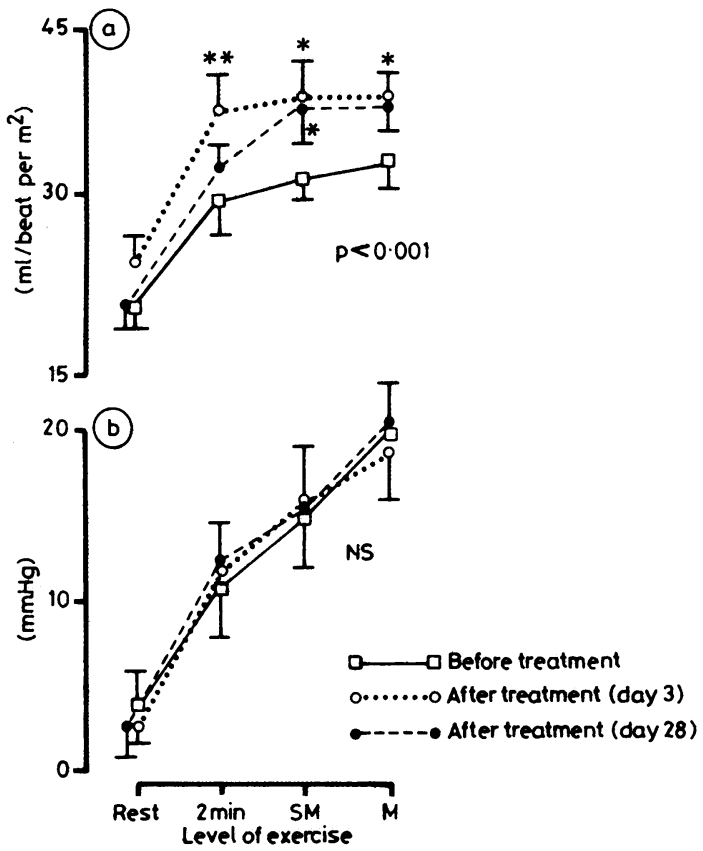

Figure Effects of milrinone on (a) stroke volume index and $(b)$ mean pubmonary capillary wedge pressure at rest, after two minutes (2 min) of exercise, at submaximal (SM) exercise, and at maximal $(M)$ exercise. Significance of difference between values after treatment and pretreatment values: $\star_{p}<0.05, \star_{p}<0.01$. Values are mean (SEM). maximal exercise was not influenced by milrinone, but systemic oxygen consumption increased significantly (Table 3). Exercise responses in other respects were unaffected.

After milrinone (day 30) - Two days after milrinone withdrawal all variables with the exception of pulmonary capillary wedge pressure reverted towards pretreatment values and were not significantly different from these values at rest or during exercise. The pulmonary capillary wedge pressure, however, was always appreciably higher than pretreatment values, and the difference was significant for the measurements at peak exercise and one minute after peak exercise (Table 2).

\section{DRUG RELATED SIDE EFFECTS}

No drug related side effects occurred during the four week treatment period. In particular, the patients experienced no gastrointestinal symptoms, and there was no thrombocytopenia $\left(<100 \times 10^{9} / 1\right)$. Mean plasma platelet concentrations $\left(\times 10^{9} / 1\right)$ in 10 patients were $270(17)$ before milrinone treatment and 217(17) after 28 days. This change was not statistically significant.

\section{Discussion}

This study has shown that milrinone treatment in heart failure is well tolerated and improves the haemodynamic response to treadmill exercise. Maximal exercise capacity is prolonged. Importantly, these beneficial effects are sustained during long term treatment. The data suggest that a drug induced increase in the perfusion of working skeletal muscle is largely responsible for the improvement in exercise tolerance.

The patients included in the present study had moderate to severe left ventricular dysfunction and were limited principally by exertional fatigue and dyspnoea. The main aim of this investigation was, therefore, to determine the effects of milrinone treatment on the haemodynamic responses to exercise. Information of this type is not currently available. Though milrinone increased cardiac index at rest, the changes in other resting measurements were not significant in contrast to the effects of acute milrinone challenge. ${ }^{14}$ The reason for this difference is not clear but might relate either to upright posture and heigh- 
tened vascular tone or to the four to five hour delay between drug dosage and haemodynamic measurements in the exercise study. During exercise milrinone produced significant increments in cardiac index without affecting left ventricular filling pressure (as measured by pulmonary capillary wedge pressure). This must reflect an improvement in left ventricular performance. The heart rate response to exercise was not influenced by the drug indicating that the improvement in cardiac index was due entirely to an increase in stroke volume index. Precise analysis of the mechanisms of these beneficial haemodynamic effects is, however, not possible, but by inference from the acute responses at rest in the same group of patients it is likely that a combination of positive inotropism and vasodilatation was responsible for the changes observed. ${ }^{14}$

The improvement in left ventricular performance during milrinone treatment was associated with an increase in systemic oxygen consumption at submaximal exercise. Previous investigators have also reported increments in exercise oxygen consumption in response to milrinone. 11 Systemic oxygen consumption is determined by cardiac output and oxygen extraction. Since the oxygen extraction of metabolising tissues is not impaired in patients with heart disease changes in systemic oxygen consumption usually reflect changes in cardiac output or, more precisely, changes in perfusion of the metabolising tissues. ${ }^{16}$ During exercise the increased oxygen demand derives primarily from the extra requirements of working skeletal muscle. Thus our finding of a milrinone induced rise in systemic oxygen consumption suggests that the drug did not significantly alter the physiological distribution of arterial blood flow during exercise and that the increase in cardiac output was delivered where it was needed-to working skeletal muscle. This contrasts with the effects of vasodilators that do not always increase oxygen consumption or maximal exercise capacity in patients with heart failure. ${ }^{1718}$ In a recent study with felodipine (a vasodilating calcium antagonist) using a similar protocol we found a reduction in systemic arteriovenous oxygen difference at submaximal exercise such that oxygen consumption was unaffected despite increments in cardiac output. ${ }^{19}$ It was argued from these data that felodipine, like hydralazine and prazosin, ${ }^{18}$ might redistribute flow away from skeletal muscle during exercise. Milrinone does not share this potentially adverse effect and may, therefore, be of greater therapeutic value than vasodilators since systemic oxygen consumption is a major predictor of functional capacity in patients with heart failure. ${ }^{20}$

The beneficial effects of milrinone on exercise cardiac index and oxygen consumption must have accounted in part for the improvement in exercise capacity measured in the present study. Previous investigators have reported a similar symptomatic improvement during milrinone treatment in heart failure. ${ }^{1012}$ Drug withdrawal, however, did not cause a significant deterioration in exercise capacity despite return of cardiac index and oxygen consumption towards control values. Thus other factors, apart from the direct effects of milrinone, must have influenced the changes in maximal exercise capacity observed in the present study. The importance of training and patient motivation, for example, is impossible to quantify, and this inevitably limits the sensitivity of serial exercise testing as a measure of drug induced changes in left ventricular function. ${ }^{21}$ More useful information is provided by direct haemodynamic measurements and gas analysis. Only in a randomised controlled study, however, can the independent effect of milrinone on exercise capacity be properly evaluated.

The natural history of congestive heart failure involves a variably progressive deterioration in left ventricular function that is often difficult to distinguish from drug tolerance in studies of this type. For this reason a four week treatment period was chosen since baseline cardiac status would not be expected to change appreciably during this relatively short period. Our data show that the improvement in exercise haemodynamics produced by milrinone was sustained during four weeks' treatment without evidence of tachyphylaxis. This finding, which accords with that of other investigators, ${ }^{10-12}$ is clearly of clinical importance for the long term management of heart failure and may distinguish milrinone from some other drugs commonly used in this condition. ${ }^{2-4}$

Two days after milrinone had been withdrawn exercise haemodynamic variables returned towards control values confirming that the changes observed during treatment were a direct result of the drug intervention. Pulmonary capillary wedge pressure, however, which had not changed during treatment, was higher than control values after drug withdrawal even though diuretic dosage was constant throughout the study. The difference was significant only at peak exercise and one minute after peak exercise and may simply reflect the extra stress, compared with that before treatment, caused by the patients' endeavours to equal the peak exercise time achieved during milrinone treatment. (The potential influence of patient motivation on exercise tolerance has already been discussed.) Alternatively, the finding could indicate a deterioration in left ventricular function to a level worse than that before treatment. Deterioration of this type has been reported after amrinone withdrawal in long term studies 22 (though this could not be confirmed in a larger placebo controlled study ${ }^{7}$ ), and, more recently, Sinoway et al observed a similar 
response to milrinone withdrawal. ${ }^{10}$ These authors attributed the phenomenon to progression of underlying disease even though the average treatment period was only seven weeks. Our patients were treated for only four weeks-a period that resulted in no measurable deterioration in baseline left ventricular function in a similar group of patients treated with felodipine. ${ }^{19}$ The possibility remains, therefore, that milrinone itself is responsible for this adverse effect. Increments in myocardial oxygen demand seen on acute milrinone challenge ${ }^{14}$ would be a potential mechanism for an accelerated decline in baseline functional status as has been suggested on theoretical grounds with other inotropic agents. ${ }^{23}$ More work is required to resolve this issue.

An important finding in the present study was the absence of drug related side effects. Though the haemodynamic effects of milrinone are in most respects similar to those of its parent compound amrinone the toxic properties of amrinone, particularly thrombocytopenia and gastrointestinal disturbance, have limited its clinical application. ${ }^{8}$ Our patients tolerated milrinone without complaint and plasma platelet concentrations were well maintained. Thus the beneficial haemodynamic effects of milrinone are combined with a high level of patient acceptability. These properties suggest a potentially useful role for milrinone treatment in the long term management of congestive heart failure. Confirmation of this role, however, must await the results of randomised controlled studies.

\section{References}

1 Fleg JL, Gottlieb SH, Lakatta EG. Is digoxin really important in treatment of compensated heart failure? A placebo-controlled crossover study in patients with sinus rhythm. Am f Med 1982; 73: 244-50.

2 Packer M, Meller J, Gorlin R, Herman MV. Hemodynamic and clinical tachyphylaxis to prazosinmediated afterload reduction in severe chronic heart failure. Circulation 1979; 59: 531-9.

3 Colucci WS, Alexander RW, Williams GH, et al. Decreased lymphocyte beta-adrenergic receptor density in patients with heart failure and tolerance to the betaadrenergic agonist pirbuterol. $N$ Engl f Med 1981; 305: 185-90.

4 Roubin GS, Choong CYP, Devenish-Mears S, et al. Beta-adrenergic stimulation of the failing ventricle: a double-blind, randomized trial of sustained oral therapy with prenalterol. Circulation 1984; 69: 955-62.

5 Benotti JR, Grossman W, Braunwald E, Carabello BA. Effects of amrinone on myocardial energy metabolism and hemodynamics in patients with severe congestive heart failure due to coronary artery disease. Circulation 1980; 62: 28-34.

6 Siegel L, Strom J, Fein E, Wexler J, Maskin L, Le Jemtel $\mathrm{T}$. Long-term effect of oral amrinone in heart failure. Improvement in exercise performance despite declining left ventricular pump function [Abstract]. $\mathrm{Am}$ f Cardiol 1981; 47: 428.
7 Dibianco R, Shabetai R, Silverman BD, Leier CV, Benotti JR. Oral amrinone for the treatment of chronic congestive heart failure: results of a multicentre randomised double blind and placebo controlled withdrawal study. FACC 1984; 4: 855-66.

8 Wilmshurst PT, Webb-Peploe MM. Side effects of amrinone therapy. Br Heart $\mathcal{F}$ 1983; 49: 447-51.

9 Alousi AA, Stankus GP, Stuart JC, Walton LH. Characterization of the cardiotonic effects of milrinone, a new and potent cardiac bipyridine, on isolated tissues from several animal species. 7 Cardiovasc Pharmacol 1983; 5: 804-11.

10 Sinoway LS, Maskin CS, Chadwick B, Forman R, Sonnenblick EH, Le Jemtel TH. Long-term therapy with a new cardiotonic agent, W1N 47203: drug-dependent improvement in cardiac performance and progression of the underlying disease. $\mathcal{F}$ Am Coll Cardiol 1981; 2: 32731.

11 Maskin CS, Sinoway L, Chadwick B, Sonnenblick EH, Le Jemtel TH. Sustained hemodynamic and clinical effects of a new cardiotonic agent, W1N 47203, in patients with severe congestive heart failure. Circulation 1981; 67: 1065-70.

12 Baim DS, McDowell AV, Cherniles J, et al. Evaluation of a new bipyridine inotropic agent-milrinone-in patients with severe congestive heart failure. $N$ Engl $\mathcal{f}$ Med 1983; 309: 748-56.

13 Franciosa JA, Weber KT, Levine TB, et al. Hydralazine in the long-term treatment of chronic heart failure: lack of difference from placebo. Am Heart $\mathcal{F}$ 1982; 104: 58794.

14 Timmis AD, Smyth P, Monaghan M, et al. Milrinone in heart failure. Acute effects on left ventricular systolic function and myocardial metabolism. $\mathrm{Br}$ Heart $\mathcal{F}$ 1985; 54: $36-41$.

15 Wallenstein S, Zucker CL, Fleiss JL. Some statistical methods useful in circulation research. Circ Res 1980; 47: 1-9.

16 Weber KT, Kinasewitz GT, Janicki JS, Fishman AP. Oxygen utilization and ventilation during exercise in patients with chronic cardiac failure. Circulation 1982; 65: 1213-23.

17 Franciosa JA, Cohn JN. Effect of isosorbide dinitrate on response to submaximal and maximal exercise in patients with congestive heart failure. Am $\mathcal{F}$ Cardiol 1979; 43: $1009-14$.

18 Rubin SA, Chatteriee K, Parmley WW. Metabolic assessment of exercise in chronic heart failure patients treated with short-term vasodilators. Circulation 1980; 61: $543-8$.

19 Timmis AD, Smyth P, Kenny JF, Campbell S, Jewitt DE. Effects of vasodilator therapy with felodipine on haemodynamic responses to treadmill exercise in congestive heart failure. Br Heart $\mathcal{f}$ 1984; 52: 314-20.

20 Franciosa JA. Functional capacity of patients with chronic left ventricular failure. Am $\mathcal{F}$ Med 1979; 67: 460-6.

21 Benge W, Litchfield RL, Marcus ML. Exercise capacity in patients with severe left ventricular dysfunction. Circulation 1980; 61: 955-9.

22 Maskin CS, Forman R, Klein NA, Sonnenblick EH, Le Jemtel TH. Long-term amrinone therapy in patients with severe heart failure. Am F Med 1982; 72: 113-8.

23 Katz AM. A new inotropic drug: its promise and a caution. N Engl f Med 1978; 299: 1409-10. 